1992-12-01

\title{
Diffraction Properties of Stratified Volume Holographic Optical Elements
}

Gregory P. Nordin

nordin@byu.edu

R. V. Johnson

A. R. Tanguay

Follow this and additional works at: https://scholarsarchive.byu.edu/facpub

Part of the Electrical and Computer Engineering Commons

\section{Original Publication Citation}

G. P. Nordin, R. V. Johnson, and A. R. Tanguay, Jr. "Diffraction Properties of Stratified Volume Holographic Optical Elements," J. Opt. Soc. Am. A, 9(12), pp. 226-2217 (1992)

\section{BYU ScholarsArchive Citation}

Nordin, Gregory P.; Johnson, R. V.; and Tanguay, A. R., "Diffraction Properties of Stratified Volume Holographic Optical Elements" (1992). Faculty Publications. 1180.

https://scholarsarchive.byu.edu/facpub/1180 


\title{
Diffraction properties of stratified volume holographic optical elements
}

\author{
Gregory P. Nordin, ${ }^{*}$ Richard V. Johnson, ${ }^{\dagger}$ and Armand R. Tanguay, Jr. \\ Optical Materials and Devices Laboratory and Center for Photonic Technology, University of Southern California, \\ Los Angeles, California 90089-0483
}

Received May 28, 1992; accepted July 13, 1992

\begin{abstract}
We present a unified treatment of the diffraction properties of stratified volume holographic optical elements (SVHOE's). We show that the relative phasing of the diffraction orders as they propagate from layer to layer gives rise to a unique notched diffraction response of the +1 order (for the case of Bragg incidence) as a function of the normalized buffer-layer thickness, the grating spatial frequency, and the readout wavelength. For certain combinations of these parameters Bragg diffraction behavior characteristic of volume holographic optical elements (VHOE's) is observed, whereas for other combinations pure Raman-Nath behavior periodically recurs. By using these same relative-phasing arguments, the principal features of the periodic angular sensitivity of the +1 and -1 orders can be predicted. In addition to examining the fundamental aspects of SVHOE diffraction behavior, we discuss several possible applications, including optical array generation, spatial frequency filtering, and wavelength notch filtering. With the use of the SVHOE concept, holographic materials with otherwise exemplary characteristics that are currently available only in thin-film form can be used in structures designed either to access unique SVHOE diffraction properties or to emulate conventional VHOE's.
\end{abstract}

\section{INTRODUCTION}

Volume holographic optical elements (VHOE's) are important in many areas of optical information processing and computing. Lately they have received wide attention as potential interconnection media for optical neural networks, ${ }^{1-7}$ in part because the angular selectivity of the Bragg diffraction process allows large numbers of interconnection gratings to be multiplexed within the same volume. Angular multiplexing of many independent holograms typically requires relatively thick holographic media. ${ }^{8,9}$ However, many currently used volume holographic materials, such as dichromated gelatin, silver halide photographic emulsions, Polaroid's DMP-128, and DuPont's Omnidex ${ }^{\mathrm{TM}}$ family of photopolymers, are available only as thin films with typical thicknesses of at most tens of micrometers. In addition, some potential new holographic media (such as multiple-quantum-well semiconductor structures $)^{10,11}$ are restricted to thicknesses that altogether preclude operation as traditional volume (i.e., Bragg) holographic optical elements.

Some of the restrictions imposed by the relatively limited thicknesses of these materials can be removed by using stratified volume holographic optical elements (SVHOE's). ${ }^{12-15}$ SVHOE's comprise a recently proposed class of novel diffraction structures in which multiple layers of a thin holographic material are interleaved with optically homogeneous buffer layers (as shown in Fig. 1). In this paper we consider the diffraction properties of SVHOE structures in which the individual holographic layers each operate within the Raman-Nath diffraction regime. The thin holographic layers perform the function of optical modulation (phase and/or amplitude), whereas the buffer regions allow diffraction to occur between the modulation layers. Although each modulation layer acts individually as a thin grating, the incorporation of buffer layers (diffraction-only regions) allows the SVHOE structure as a whole to emulate the properties of volume media in which optical modulation occurs concurrently with the diffraction process during propagation of a readout beam through the medium. In addition, the physical separation of the modulation and diffraction processes in SVHOE's allows the SVHOE's to exhibit unique diffraction properties that are unavailable with the use of conventional thin (single-layer) or bulk holographic media. By using these unique properties, one can create novel devices for a variety of applications such as array generation and wavelength notch filtering. Depending on the desired application, SVHOE's can be designed to function as traditional VHOE's or as unique diffraction devices. We also note that the SVHOE concept suggests the possibility of fabricating volume computer-generated holograms (VCGH's) by stacking layers of planar computer-generated holograms. A further option is active (optical or electrical) control of each modulation layer for appropriate modulation layer media. ${ }^{13,16}$

In this paper we first review previous work on systems of stacked gratings and then discuss the fundamental diffraction properties of SVHOE's and their physical origins. We consider SVHOE's composed of multiple layers of thin (in the Raman-Nath diffraction sense) holographic media in which the physical separation of the layers is constant for all layers. We treat the case in which a single unslanted sinusoidal phase grating is recorded in all the layers by a pair of recording beams as shown in Fig. 1 . Using numerical modeling results and analytical arguments, we show that periodic Bragg diffraction responses arise for both the +1 and -1 diffraction orders and discuss their angular dependence. The dependence of SVHOE diffraction properties on buffer-layer thickness, number of modulation layers, total device thickness, and total grating strength is derived and explained. In addi- 


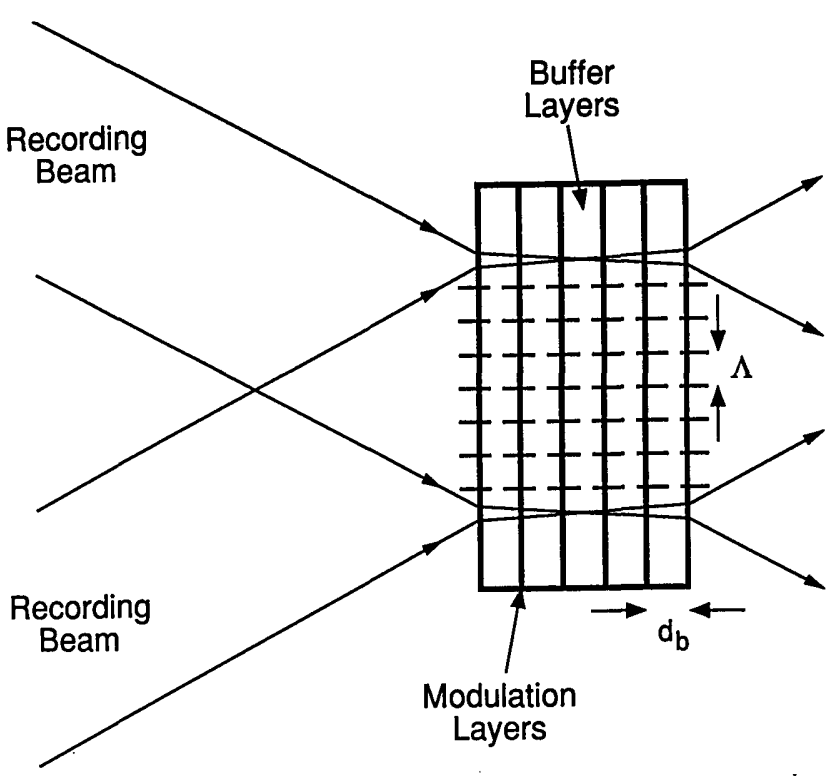

Fig. 1. Schematic diagram of the SVHOE structure, in which modulation layers composed of a thin holographic recording material are interleaved with optically homogeneous buffer layers.

tion, we show further novel SVHOE diffraction behavior as a function of the normalized buffer-layer thickness $Q_{b}$, defined as

$$
Q_{b}=2 \pi \lambda_{0} d_{b} /\left(n \Lambda^{2}\right),
$$

in which $\lambda_{0}$ is the free-space wavelength of the readout beam, $d_{b}$ is the buffer-layer thickness, $n$ is the refractive index of the buffer layers, and $\Lambda$ is the grating period of the grating recorded in the modulation layers. We show that at Bragg incidence the first diffraction order exhibits a novel periodic dependence on the normalized bufferlayer thickness $Q_{b}$, which we explain in terms of the relative phasing of the diffracted orders as they propagate from layer to layer. We then show how this behavior leads to a notched diffraction response of the first order as a function of both the readout wavelength and the grating spatial frequency. Possible applications of the novel SVHOE diffraction behavior discussed in this paper include optical array generation ${ }^{13}$ and wavelength notch filtering. ${ }^{15}$

The numerical analyses of SVHOE diffraction properties discussed in Sections 3 and 4 were performed by using the optical beam propagation method (BPM). ${ }^{17}$ A discussion of this method and its application to grating diffraction problems can be found in Refs. 18 and 19. Throughout the paper we assume that the usual smallangle approximations are valid and so limit the analysis to paraxial propagation.

\section{REVIEW OF PREVIOUS WORK}

The physical separation of the diffraction and modulation processes as embodied in the SVHOE structure was originally suggested to several of us $^{13}$ by the algorithmic separation of those processes in the optical beam propagation method, which is a numerical modeling tool used to analyze optical propagation through various types of media. ${ }^{17-21}$ As indicated by application of BPM to diffraction-grating problems, the demonstration of pronounced Bragg diffraction behavior requires surpris- ingly few modulation layers. ${ }^{19}$ Johnson and Tanguay showed (using BPM) that the SVHOE +1 order exhibits a periodic angular sensitivity and verified this behavior experimentally. ${ }^{13}$ The notched diffraction response of the +1 order as a function of the normalized buffer-layer thickness was first discussed in Ref. 15. We present in this paper a unified treatment of these phenomena and their origins as well as new results on the behavior of the -1 order.

Stacks of more than one holographic layer have been proposed previously in several contexts, including optical memories in which each layer stores a page of information. ${ }^{22-25}$ In this particular application, a readout technique is required that minimizes interlayer interactions such that each layer can be addressed independently. In the absence of such techniques, several studies suggest severe information readout difficulties. ${ }^{26-29}$ In the SVHOE concept, it is precisely the interlayer interactions and their consequent effects on diffraction behavior that give SVHOE's the ability to emulate VHOE diffraction and that are also responsible for novel SVHOE properties.

Another earlier proposal concerning the use of multiple layers of gratings was made by De Bitetto, ${ }^{30}$ who considered what he termed a "pile-of-gratings" (formed by stacking sheets of inexpensive plastic gratings) to increase the diffraction efficiency of a diffracted image beam. His analysis considered only incoherent addition of the intensities of the diffracted orders from each layer, which he justified by arguing that the phase relationship between orders in his system was destroyed by various nonuniformities and lack of grating alignment from layer to layer. These arguments do not hold for the SVHOE concept discussed in this paper. We are concerned herein only with coherent addition of the diffracted orders from each modulation layer.

Structures composed of two sequential thin grating layers, which in some sense are the simplest case of the SVHOE concept, have been studied extensively in the literature. ${ }^{31-47}$ Studies have been made of two-layer structures in which both layers are fixed gratings, one layer is a fixed grating and the other is an acoustic grating, or both layers are acoustic gratings. Both bulk and surface acoustic waves (SAW's) have been used.

The earliest investigation of two-layer grating structures was performed in 1960 by Hargrove, ${ }^{31}$ who reported preliminary experimental studies of diffraction by spatially separated bulk acoustic waves. His results were subsequently explained by using thin-grating diffraction theory applied to two sequentially arranged gratings. ${ }^{32}$ For readout at normal incidence with a collimated readout beam, he found that the first-order diffraction efficiency varied sinusoidally (with period $Q_{b} / \pi$ ) as a function of the normalized separation of the ultrasonic gratings $Q_{b}$. Malysh et $a l . .^{34}$ demonstrated a similar periodic dependence of the first-order diffraction efficiency on grating separation for readout at normal incidence. The gratings that they investigated were formed by the film-air and filmsubstrate interfaces of a thin film deposited upon a corrugated substrate. In this paper we show a novel periodic notched diffraction response of the first diffraction order as a function of the normalized buffer-layer thickness $Q_{b}$ (with period $Q_{b} / 2 \pi$ ) for readout at Bragg incidence for SVHOE's with arbitrary numbers of layers. 
Zel'dovich et $a l .{ }^{42}$ have considered two-layer structures in which each layer is composed of a fixed grating. They showed analytically that the first diffraction order varies periodically as a function of readout-beam incidence angle, which also has been demonstrated experimentally. ${ }^{43} \mathrm{~A}$ major motivation for their work was the achievement of higher diffraction efficiencies with the use of two-layer gratings than can be achieved in single-layer gratings. This is of interest for holographic recording applications in which large diffraction efficiencies are desired. Other diffraction orders (besides the first) also have been found to exhibit a periodic angular response. This has resulted in the proposal of using both two- and four-layer grating structures as optical analog-to-digital converters. ${ }^{44,45}$

Kujawinska ${ }^{48}$ recently theoretically examined optical readout of a series of sequentially arranged quasi-periodic gratings illuminated by quasi-spherical wave fronts. His treatment, however, is essentially a restatement of the algorithm embodied in the optical BPM. ${ }^{17}$ Kujawinska has applied this methodology to Talbot and grating-shearing interferometers ${ }^{46}$ that incorporate two grating layers.

Yakimovich ${ }^{49}$ has discussed the diffraction efficiency and angular selectivity properties of thick (i.e., Bragg regime) modulation layers interleaved with homogeneous buffer layers. His analysis is based on a coupled-wave approach in which only the 0 and +1 orders are retained. Because we treat the case in which the modulation layers are composed of thin (Raman-Nath) gratings, all significant forward-propagating orders are retained in our numerical calculations (typically up to at least the \pm 8 th orders). As will be seen below, observation of notched first-order diffraction behavior as a function of the normalized buffer-layer thickness requires consideration of the higher orders.

Multiple layers of gratings composed of conducting wires or grids have been examined by several authors, principally as antireflection elements or high-reflectivity mirrors for the far-IR to microwave wavelength regions. ${ }^{50-52}$ These applications require that the grating period be of the order of or smaller than the wavelength of the illuminating beam, and only zeroth-order (both forwardpropagated and reflected) components are typically considered in the analysis of such systems. In this paper we are concerned only with cases in which the grating period is significantly larger than the wavelength of the illuminating beam such that traditional grating diffraction properties are obtained for each modülation layer.

\section{SVHOE ANGULAR SENSITIVITY}

\section{A. +1 Diffraction Order}

A key distinguishing feature of volume compared with thin holograms is the dependence of the diffraction efficiency of the first order on the incidence angle of the readout beam. ${ }^{53}$ In this section we describe the corresponding angular properties of SVHOE diffraction and the physical origin of these properties. We concentrate initially on the properties of the first diffraction order as a function of readout beam incidence angle, as shown in Fig. 2. Note that as the incidence angle of the readout beam is changed the diffracted orders rotate with the incident beam such that they maintain the same angular position with respect to the zeroth order. For purposes of our discussion, positive angles are defined by rotation counterclockwise about an axis extending perpendicularly out of the plane of Fig. 2. Plane-wave readout illumination is assumed, and all angles are measured within the medium.

The buffer-layer thickness, total thickness, and number of modulation layers play a key role in determining the angular sensitivity of SVHOE diffraction behavior. We investigate the effects of these parameters by considering two sets of SVHOE's as shown in Fig. 3 in which either the interlayer separation (case A) or the total thickness (case B) is held constant as the number of layers is increased. A total grating strength of $2.1 \mathrm{rad}$ is assumed for each SVHOE. The grating strength $v$ is defined as

$$
v=2 \pi \Delta n D_{\bmod } / \lambda_{0},
$$

in which $\Delta n$ is the amplitude of the sinusoidal refractive-

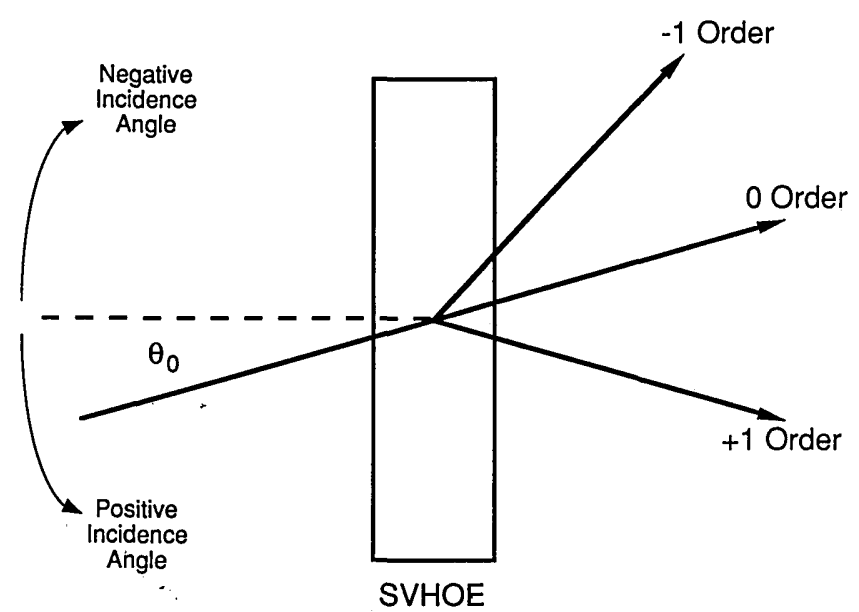

Fig. 2. Definition of the readout beam incidence angle $\theta_{0}$ relative to the SVHOE surface normal.
Case A

Constant Buffer Layer Thickness $(=150 \mu \mathrm{m})$

$\|$

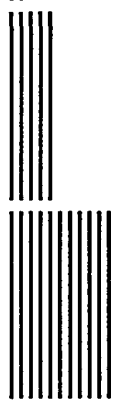

\section{Case B}

\section{Constant Total Thickness} $(=1500 \mu \mathrm{m})$

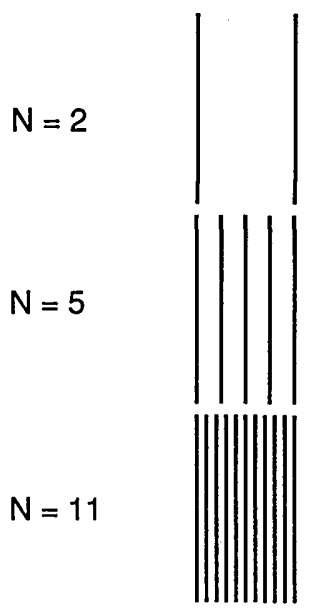

Fig. 3. Relative buffer-layer thicknesses and total thicknesses of two sets of SVHOE's. The SVHOE's in case A all have the same buffer-layer thickness $(150 \mu \mathrm{m})$, whereas the SVHOE's in case B have the same total thickness $(1500 \mu \mathrm{m})$. The angular sensitivities of the first diffraction orders for these sets of SVHOE's are compared in Figs. 4 and 5. Note that the 11-layer SVHOE has the same buffer-layer thickness and total thickness in both cases. 


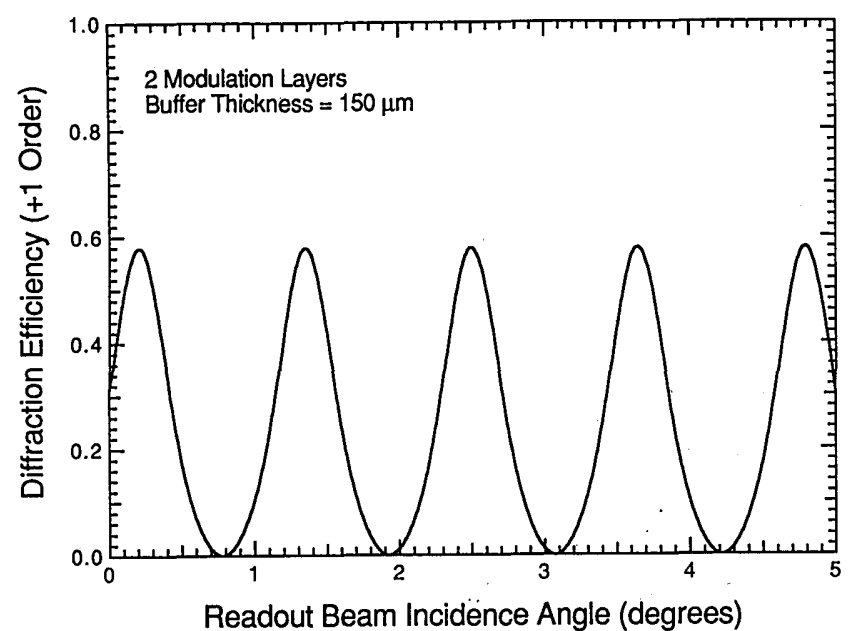

(a)

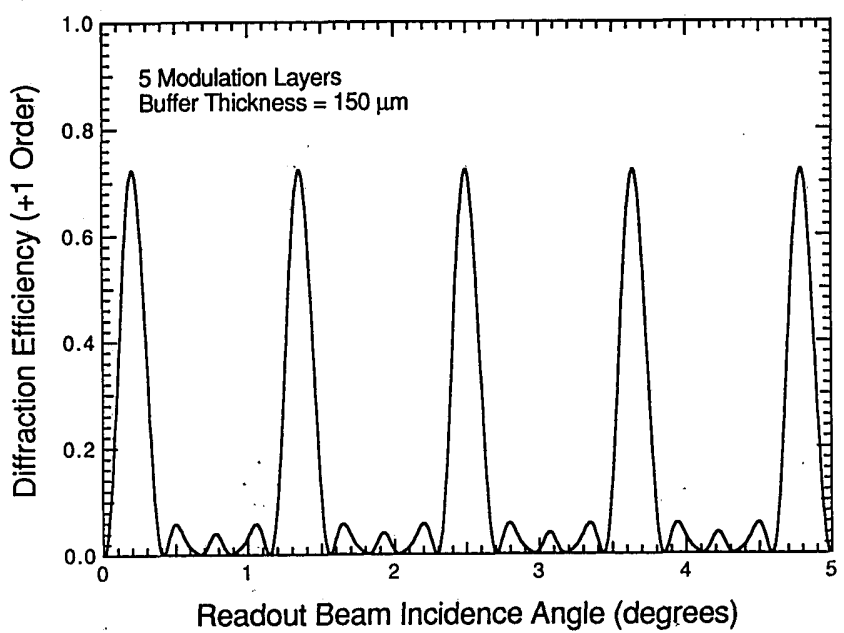

(b)

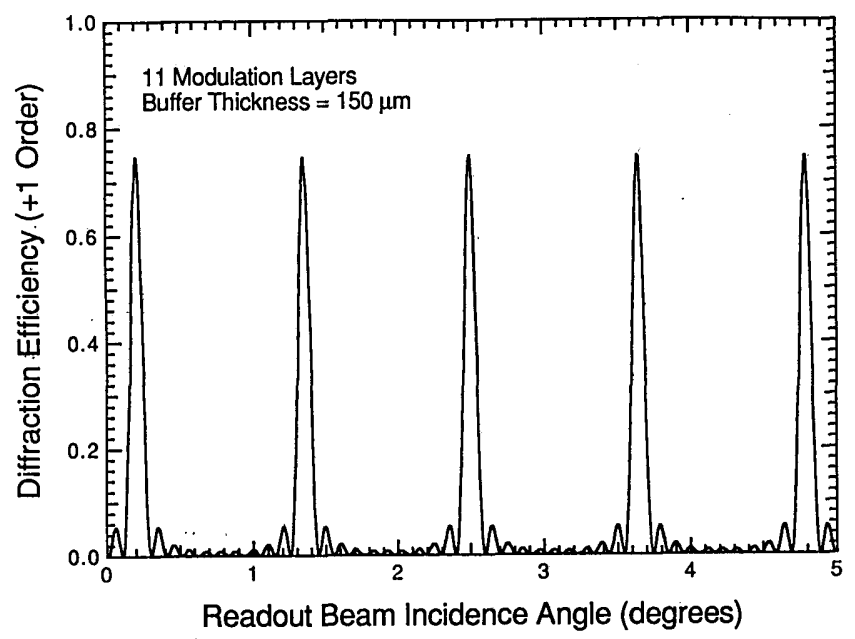

(c)

Fig. 4. First-order diffraction efficiency as a function of readout beam incidence angle for the 2-, 5-, and 11-layer [(a), (b), and (c), respectively] SVHOE's of case A.

index modulation and $D_{\text {mod }}$ is the total thickness of the modulation layers (i.e., $D_{\text {mod }}=N d_{\text {mod }}$, in which $N$ is the number of modulation layers and $\dot{d}_{\text {mod }}$ is the thickness of an individual layer). The readout wavelength is chosen as $0.89 \mu \mathrm{m}$. The refractive index of the buffer and modula- tion layers is 3.4 , and the grating period $\Lambda$ is $3.0 \mu \mathrm{m}$. The Bragg angle $\theta_{\mathrm{B}}\left[\sim \lambda_{0} /(2 n \Lambda)\right]$ is thus $2.5^{\circ}$. In case $\mathrm{A}$ (constant buffer-layer thickness) $d_{b}=150 \mu \mathrm{m}$, whereas in case $B$ (constant total thickness) the total thickness is $1500 \mu \mathrm{m}$.

Figures 4 and 5 show the angular sensitivities of the SVHOE's for cases A and B, respectively. When the buffer-layer thickness is held constant (Fig. 4), the addition of layers decreases the angular width of the diffraction peaks because of the increase of the total SVHOE thickness. (For more than three or four modulation layers, the FWHM of each diffraction peak is essentially the same as for a VHOE of comparable thickness, namely, $\sim \Lambda / D_{t}$, in which $D_{t}$ is the total SVHOE or VHOE thickness.) The angular position of each peak, however, remains the same, indicating that the peak position depends on the buffer-layer thickness and not on the total thickness or the number of layers. ${ }^{13}$ For the two-layer SVHOE [Fig. 4(a)], the diffraction response is nearly sinusoidal. The peak diffraction efficiency is $58 \%$, which is well above the thin-grating result of $32 \%$ (at $v=2.1 \mathrm{rad}$ ). ${ }^{43}$ The diffraction behavior of the five-layer SVHOE illustrated in

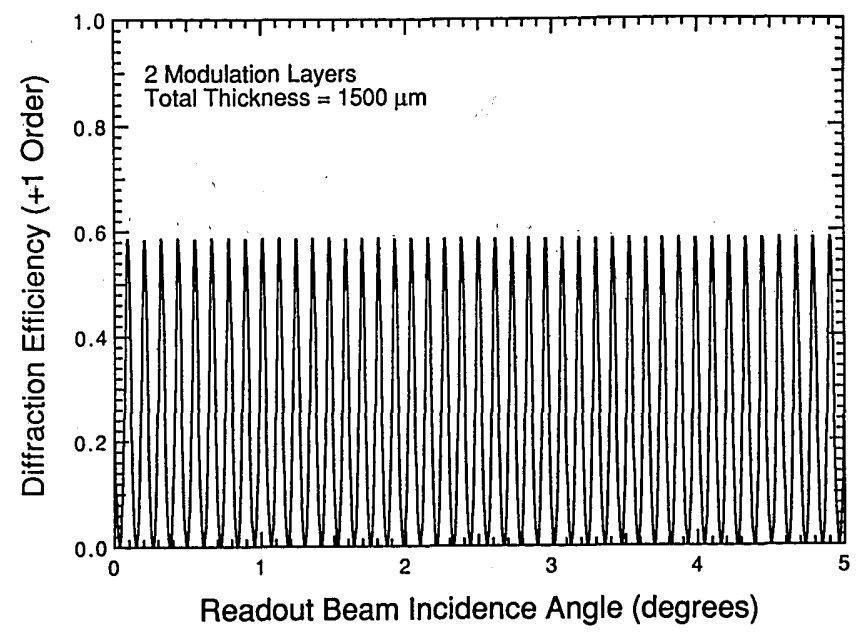

(a)

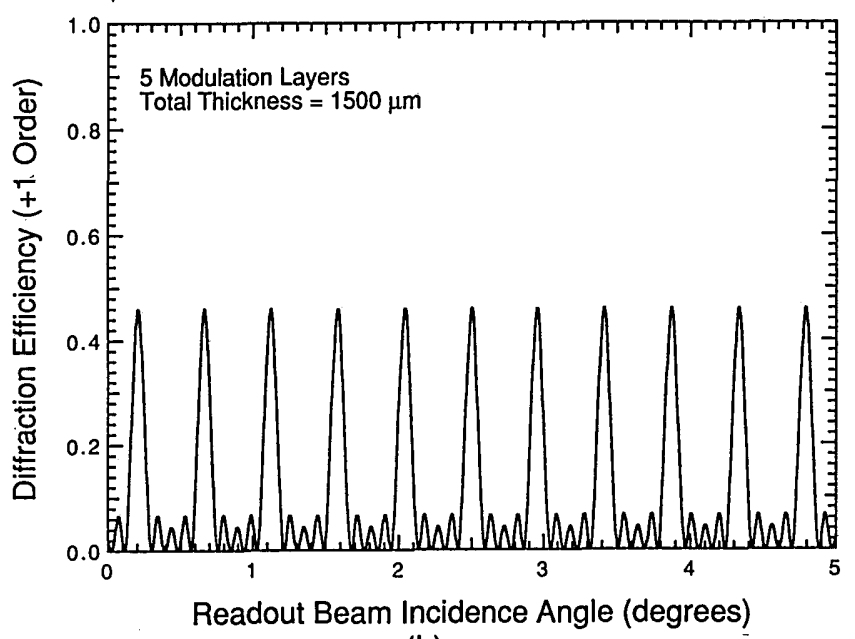

(b)

Fig. 5. First-order diffraction efficiency as a function of readout beam incidence angle for the two- and five-layer [(a) and (b), respectively] SVHOE's of case B. The angular response of the case B 11-layer SVHOE is the same as that shown in Fig. 4(c). 
Fig. 4(b) shows that relatively few modulation layers are required for one to obtain nearly the same peak diffraction efficiency as a comparable Bragg grating, which is $75 \%$. As the number of layers increases, the peak diffraction efficiency asymptotically approaches that of a grating recorded in a conventional volume medium [see Fig. 4(c) for the 11-layer case].

Figure 5(a) shows the angular response of the two-layer SVHOE representing case $B$. The angular period of the diffraction peaks is one tenth that of the two-layer SVHOE of case A because of the factor-of-10 difference in modulation-layer separation. As is shown in Fig. 5(b), the effect of increasing the number of modulation layers from two to five (while keeping the total thickness the same) is to suppress three of every four peaks observed in the angular response of the two-layer SVHOE. In general, the result of adding modulation layers when the total SVHOE thickness is held constant is to suppress $N-2$ of every $N-1$ two-layer diffraction peaks. ${ }^{13}$ The diffraction peak occurring at Bragg incidence is of course always retained, as can be seen by comparing Figs. 5(a), 5(b), and 4(c). A final point is that in case $B$ the peak diffraction efficiency of the five-layer SVHOE [Fig. 5(b)] is smaller than that of the two-layer SVHOE [Fig. 5(a)]. Increasing the number of layers in this case has actually decreased the peak diffraction efficiency. The reason for this behavior will become apparent after our discussion of the angular sensitivity of the -1 order.

The angular position of each diffraction peak may be easily derived by considering the relative phases of the zeroth and first orders after propagation between modulation layers (see Fig. 6). In a distributed bulk grating, a Bragg first-order diffraction peak occurs when the zeroth and first orders maintain the same relative optical phase throughout the medium as they are modulated by the periodic refractive-index distribution (this condition occurs at Bragg incidence). We therefore expect SVHOE's to exhibit similar Bragg-type diffraction when the zeroth and first orders have the same relative phase at each modulation layer.

We can express this condition as follows. Let $\phi_{1}$ be the phase difference between the zeroth and first orders after propagation between two modulation layers. This phase difference may be written as

$$
\begin{aligned}
\phi_{1} & =2 \pi n d_{b}\left(\cos \theta_{1}-\cos \theta_{0}\right) / \lambda_{0} \\
& \approx \pi n d_{b}\left(\theta_{0}{ }^{2}-\theta_{1}{ }^{2}\right) / \lambda_{0},
\end{aligned}
$$

in which (as shown in Fig. 6) $\theta_{0}$ is the incident angle and $\theta_{1}$ is the angle at which the +1 order is diffracted. Both $\theta_{1}$ and $\theta_{0}$ are assumed to be small. For the relative phases of the +1 and 0 orders to be the same at each modulation layer, $\phi_{1}$ must be an integer multiple of $2 \pi$. Using this condition in expression (3) and recognizing that the angle of the first diffraction order can be written as $\theta_{1}=$ $\theta_{0}-\lambda_{0} /(n \Lambda)$ (using the small-angle approximation), we solve for $\theta_{0}$ and obtain the incidence angles at which Bragg responses are expected:

$$
\begin{aligned}
\theta_{0} & =\lambda_{0} /(2 n \Lambda)+l_{1} \Lambda / d_{b} \\
& =\theta_{B}+l_{1} \Delta \theta_{p},
\end{aligned}
$$

in which $l_{1}=0, \pm 1, \pm 2, \ldots$ and $\Delta \theta_{p} \equiv \Lambda / d_{b}$.
According to Eq. (4), SVHOE diffraction peaks occur at angular intervals of $\Delta \theta_{p}$ centered about the Bragg angle. When we examine Figs. 4 and 5 , this is exactly the behavior that we observe in the BPM calculations. For the SVHOE's of case A with $\Lambda=3.0 \mu \mathrm{m}$ and $d_{b}=150 \mu \mathrm{m}$, the predicted peak separation of $1.15^{\circ}$ from Eq. (4) matches the observed separation seen in Figs. 4(a)-4(c). Similarly, in Figs. 5(a) and 5(b) the predicted and observed peak separations are also the same $\left(0.12^{\circ}\right.$ and $0.46^{\circ}$, respectively). The angular sensitivity characteristics of SVHOE's with thin modulation layers are summarized in Fig. 7.

As is noted in Ref. 13, the periodic angular response of an SVHOE in which a single grating is recorded can be used to generate a one-dimensional (1-D) array of equal amplitude beams that are evenly separated in angle by simply

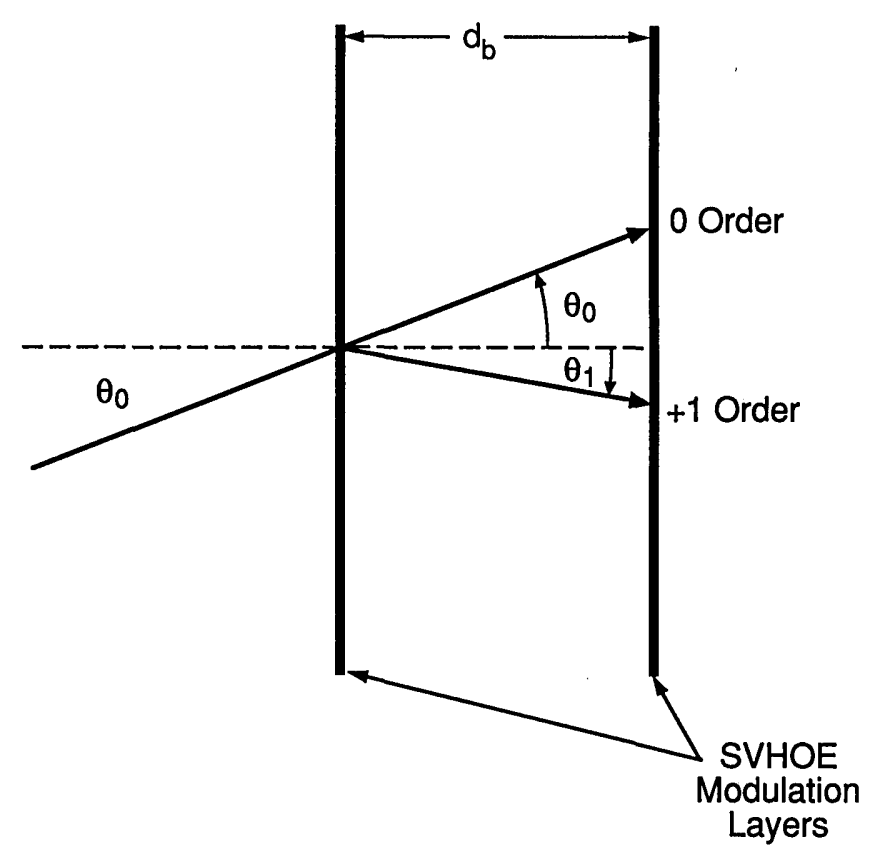

Fig. 6. Propagation of the zeroth and first orders between SVHOE layers, with an incidence angle of $\theta_{0}$. The first order propagates at an angle $\theta_{1}$ with respect to the surface normal of the SVHOE.

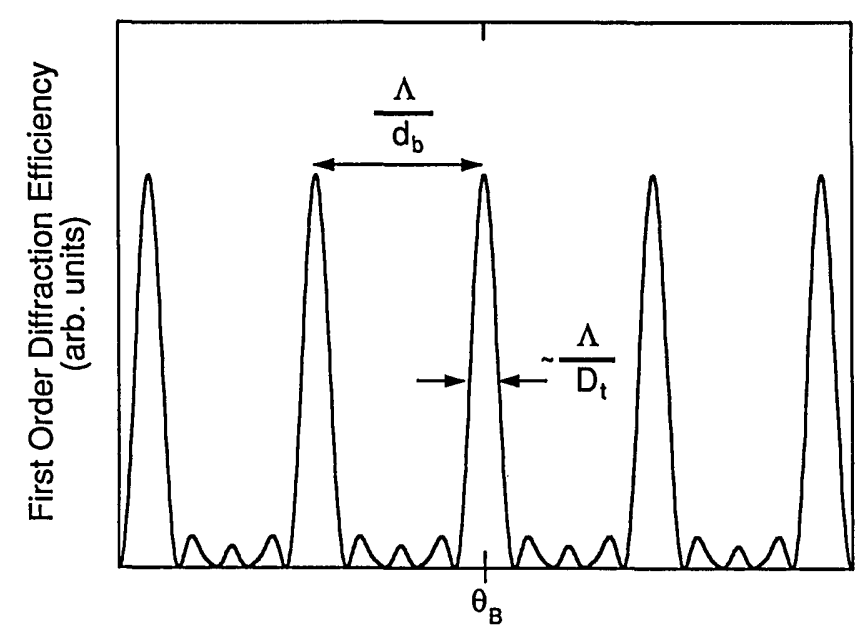

Readout Beam Incidence Angle (radians)

Fig. 7. Summary of SVHOE +1-order angular diffraction behavior. 
illuminating an SVHOE with a focused uniform readout beam. The actual number of beams that are produced is given by $d_{b} /(n \Lambda F)$, in which $F$ is the $f$-number of the illuminating beam. Large numbers of equal-amplitude beams can be generated by making the buffer-layer thickness large and the grating period and the $f$-number sufficiently small. [Note, however, that the smallest-f-number readout beam that can be used for a given grating period is $\approx 1 /\left(2 \theta_{B}\right)$ because the zeroth order and +1 orders overlap for smaller $f$-numbers.] A two-dimensional array of uniform beams can be generated by cascading two SVHOE's such that the respective grating wave vectors in the SVHOE's are orthogonal. For example, two of the twolayer SVHOE's in case B above can be used to generate an array of $175 \times 175$ beams if the readout beam is focused to $F / 1.7$ and the grating period is $1.5 \mu \mathrm{m}$ instead of $3.0 \mu \mathrm{m}$.

\section{B. -1 Diffraction Order}

For purposes of this paper, the labeling of the +1 and -1 orders as shown in Fig. 2 is maintained for all incidence angles. This scheme becomes somewhat artificial for conventional volume holographic optical elements when negative incidence angles are examined because the Bragg diffracted order is typically referred to as the +1 order rather than the -1 order. For example, consider the angular response of a grating recorded in a VHOE with the same thickness and other physical parameters as the SVHOE's of case B discussed above. As is shown in Fig. 8, we obtain the expected diffraction peak at Bragg incidence $\left(2.5^{\circ}\right)$ for the +1 order. There is naturally also a diffraction peak at $-\theta_{B}$ for what has been labeled the -1 order, which is a mirror image (about normal incidence) of the first-order diffraction response at $+\theta_{B}$.

Although seemingly trivial, the question of labeling is important in the context of SVHOE's because both +1 - and -1-order diffraction peaks can occur within any given range of incidence angles, regardless of whether positive or negative incidence angles are considered. This is illustrated in Fig. 9, in which we show the angular response (at both positive and negative incidence angles) of the +1 and -1 orders of the 11-layer SVHOE examined previously [see Fig. 4(c)]. Both +1- and -1-order angular responses occur within the angular range shown in Fig. 9. The -1-order diffraction behavior has the same physical characteristics and origins as noted above for the +1 order, except that the -1 -order peaks are centered about $-\theta_{B}$; i.e., -1 -order diffraction peaks occur at incidence angles of

$$
\theta_{0}=-\theta_{B}+l_{-1} \Delta \theta_{p},
$$

in which $l_{-1}=0, \pm 1, \pm 2, \ldots$ The +1 - and -1 -order diffraction responses are of course simply mirror images of each other about normal incidence (for unslanted gratings).

One implication of this periodic-1-order behavior is that an array generator based on the illumination of an SVHOE with a focused beam also generates a set of -1 -order beams in addition to the set of +1 -order beams as discussed at the end of Subsection 3.A. This is shown schematically in Fig. 10 for a 1-D SVHOE-based array generator. A single SVHOE used to produce a 1-D array of beams thus produces two such arrays, and a pair of cascaded SVHOE's used to generate a 2-D array produces four arrays of beams.
C. Overlap of the +1- and -1-Order Angular Sensitivities The periodicity of both the +1 - and -1-order diffraction responses is a key difference between SVHOE and VHOE diffraction properties and leads to further novel SVHOE behavior. For example, because the angular separation of

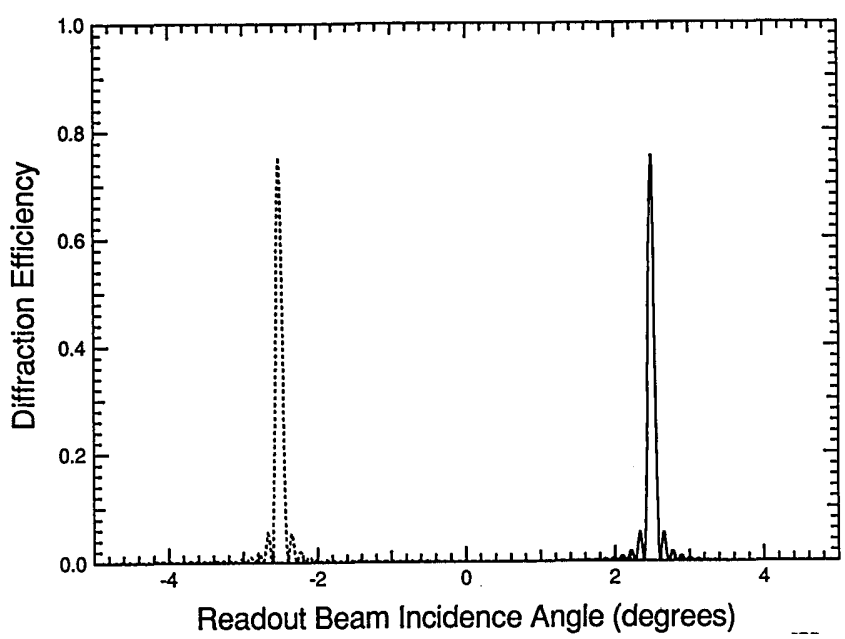

Fig. 8. Angular diffraction sensitivity of the +1 (solid curve) and -1 (dashed curve) orders of a $1500-\mu \mathrm{m}$-thick volume hologram. The grating recorded in the material has a period of $3.0 \mu \mathrm{m}$. The material is assumed to have the same refractive index (3.4) as the SVHOE's in cases A and B.

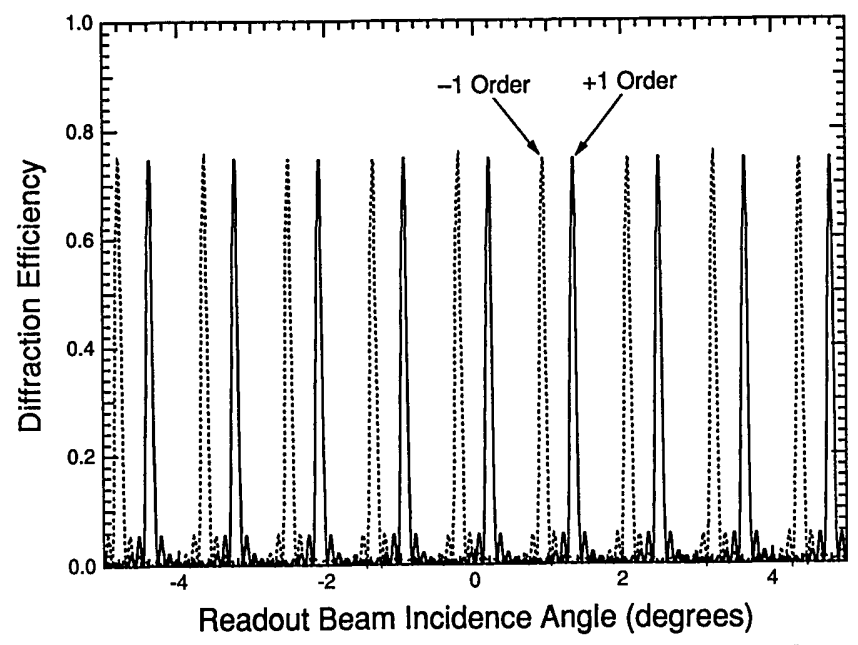

Fig. 9. Angular sensitivity of the +1 order (solid curve) and -1 order (dashed curve) of the 1500- $\mu$ m-thick 11-layer SVHOE in cases $\mathrm{A}$ and $\mathrm{B}$.

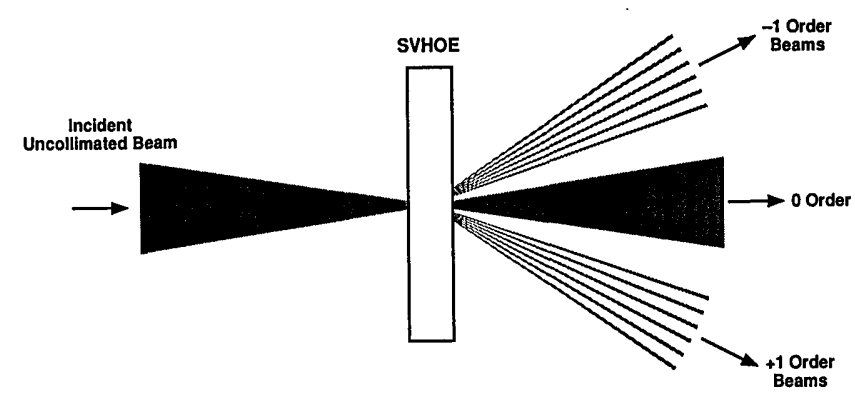

Fig. 10. Schematic diagram showing an SVHOE operating as a 1-D array generator for readout with an uncollimated beam. Two 1-D arrays of beams are generated, one associated with the +1 order and the other associated with the -1 order. 
SVHOE \pm 1 -order response peaks is dependent on the buffer-layer thickness $d_{b}$, values of $d_{b}$ can be chosen for a given grating period that cause the +1 - and -1-order diffraction peaks to occur at the same incidence angles. Exact overlap of the +1- and -1-order angular sensitivities occurs when an integer number of peak separations, $\Delta \theta_{p}$, fits exactly in the angular range between $-\theta_{B}$ and $+\theta_{B}$. We express this condition as

$$
p=2 \theta_{B} / \Delta \theta_{p}
$$

in which $p$ is an integer greater than zero. By substituting the definitions of $\theta_{B}$ and $\Delta \theta_{p}$ into Eq. (6), one may rewrite Eq. (6) as

$$
p=Q_{b} /(2 \pi)
$$

in which $Q_{b}$ is the normalized buffer-layer thickness defined in Eq. (1). According to Eq. (7), overlap of the +1and -1-order angular sensitivities occurs at integer values of the normalized buffer-layer thickness divided by $2 \pi$. In the remainder of this section we discuss the implications of this result for the angular sensitivity of SVHOE's. In Subsection 4.A we show how this angular overlap plays a role in the notched +1-order diffraction behavior exhibited by SVHOE's for readout at Bragg incidence. This curious behavior includes the occurrence of Raman-Nath diffraction efficiencies for all the diffraction orders of SVHOE's having integer values of $Q_{b} / 2 \pi$.

We illustrate several of the effects of \pm 1 -order angular sensitivity overlap in Figs. 11(a) and 11(b). Here we show both the +1- and the -1-order angular sensitivities of a five-layer SVHOE in which ' $Q_{b} / 2 \pi$ is 4.0 and 4.5 , respectively. The total grating strength is $2.0 \mathrm{rad}$. When $Q_{b} / 2 \pi$ is an integer [Fig. 11(a)], the +1 and -1 orders have identical angular sensitivities. In the presence of large grating strengths, competition occurs between the +1 and -1 orders for energy from the zeroth order at what would otherwise be Bragg diffraction peaks for each order. The consequence is reduced diffraction efficiency in both the +1 - and the -1-order peaks relative to cases in which the \pm 1 -order diffraction peaks are separated. This is illustrated by comparing Figs. 11(a) and 11(b). If SVHOE's are used to replace a thin grating to achieve higher diffraction efficiency in applications in which a large grating strength is required, attention must be paid to the choice of buffer-layer thickness and grating period such that overlap of the +1- and -1-order diffraction responses does not occur. Otherwise, the peak diffraction efficiency can be reduced rather than enhanced. This is illustrated by comparing the two- and five-layer SVHOE diffraction responses shown in Figs. 5(a) and 5(b). The reason for the reduced diffraction efficiency of the five-layer SVHOE (as noted above) is that partial overlap of the \pm 1 -order angular sensitivities is present $\left(Q_{b} / 2 \pi\right.$ is 10.9 -nearly an integer).

The relationship between the incidence angles at which +1 - and -1-order SVHOE diffraction peaks occur and the normalized buffer-layer thickness is further illustrated in Fig. 12. The horizontal axis represents the normalized incidence angle, defined as $\alpha=\theta_{0} / 2 \theta_{B}$, whereas the vertical axis represents the normalized buffer-layer thickness $Q_{b}$ divided by $2 \pi$. The solid curves indicate the position of the +1 -order diffraction peaks as a function of these two parameters, whereas the -1-order peak positions are indicated by dashed curves. As expected, there is a diffraction peak at Bragg incidence $(\alpha=1 / 2)$ for the +1 order and at $-\theta_{B}(\alpha=-1 / 2)$ for the -1 order for all values of the normalized buffer-layer thickness. At integer values of $Q_{b} / 2 \pi$, the angular positions of the +1 and the -1 orders coincide.

As discussed above, the peak positions of the \pm 1 orders are independent of the number of SVHOE modulation layers at constant buffer-layer thickness. Figure 12 therefore holds for all SVHOE's, regardless of whether they have two or one hundred layers. For example, the angular peak positions shown in Figs. 11(a) and 11(b) for a five-layer SVHOE are predicted exactly by Fig. 12 at the appropriate normalized buffer-layer thicknesses. Note also that the period of the diffraction behavior observed by Hargrove $e t a l .^{32}$ for a two-layer structure is easily seen in Fig. 12; namely, at normal incidence the +1 -order peaks occur at intervals of $2\left(Q_{b} / 2 \pi\right)$ as a function of the normalized buffer-layer thickness.

A further interesting result is that for a given SVHOE the fractional part of $Q_{b} / 2 \pi$ indicates the relative angular position of any -1-order peak between its two adjacent

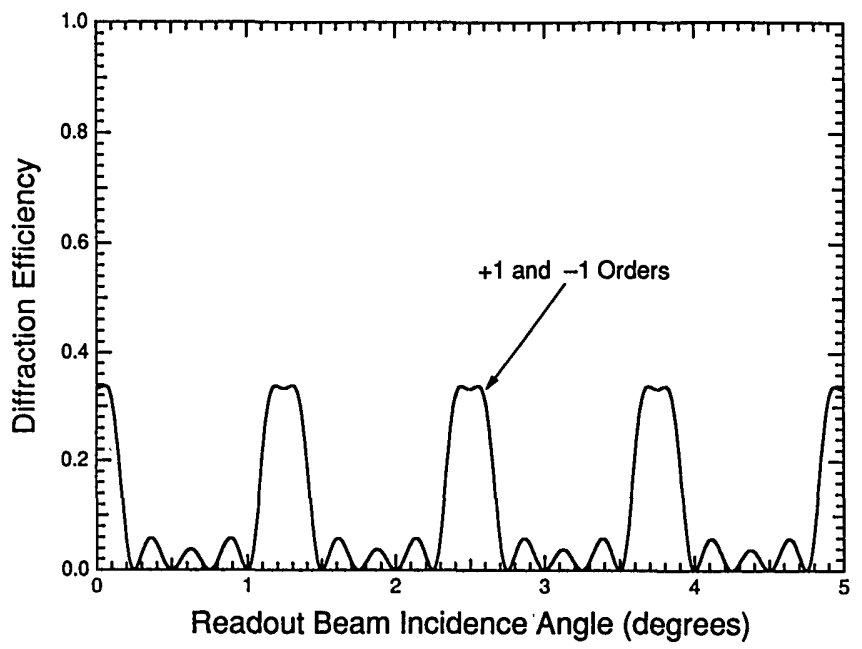

(a)

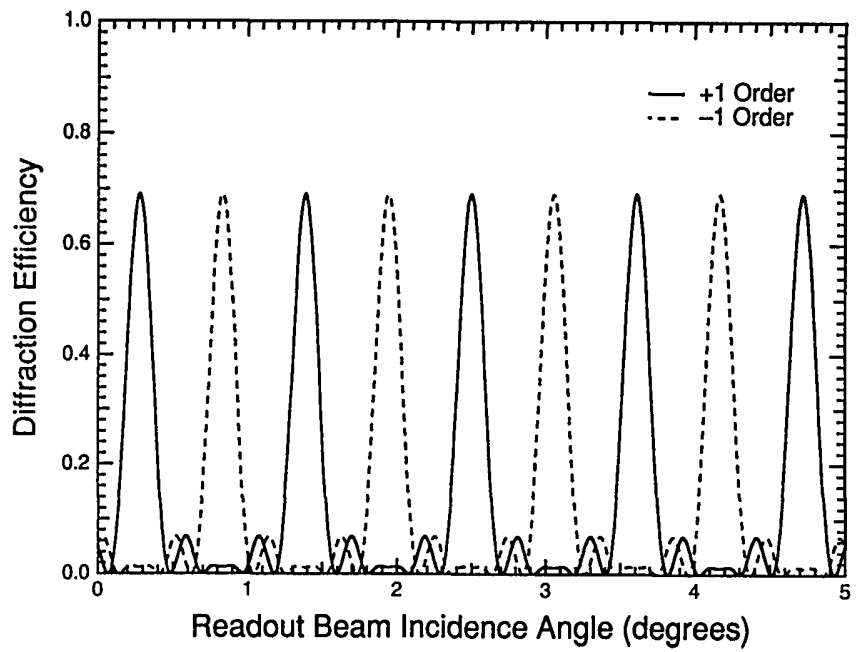

(b)

Fig. 11. Angular sensitivity of the \pm 1 orders for a five-layer SVHOE for (a) $Q_{b} / 2 \pi=4.0$ and (b) $Q_{b} / 2 \pi=4.5$. 


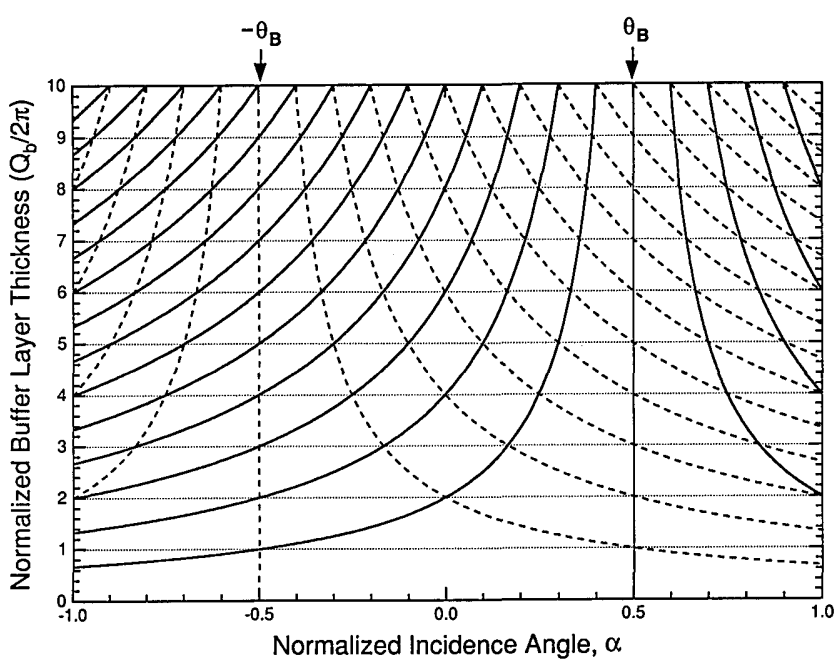

Fig. 12. Positions of SVHOE +1-order and -1-order diffraction peaks (solid and dashed curves, respectively) as a function of the normalized incidence angle $\alpha$ and the normalized buffer-layer thickness $Q_{b} / 2 \pi$. At integer values of $Q_{b} / 2 \pi$, the \pm 1 -order diffraction peaks occur at the same incidence angles.

+1-order peaks (and vice versa). For example, as is seen in Fig. 11(b), each -1-order peak is exactly halfway between its adjacent +1 -order peaks when $Q_{b} / 2 \pi=4.5$. Similarly, the integer part of $\left(Q_{b} / 2 \pi+1\right)$ gives the number of +1 - and -1 -order diffraction peaks in the angular interval between $\pm \theta_{B}$. Thus knowing the normalized buffer-layer thickness of a particular SVHOE immediately gives insight into the relative angular spacing and number of \pm 1 -order diffraction peaks that occur for readout at incidence angles between the positive and negative Bragg angles.

\section{VHOE Emulation}

The behavior of SVHOE +1- and -1-order angular sensitivity is useful for explaining many of the novel diffraction effects exhibited by SVHOE's as well as the regimes in which SVHOE's can emulate the properties of conventional VHOE's. As we pointed out in Section 1, SVHOE emulation of VHOE characteristics offers the opportunity of using thin holographic materials in applications that require diffraction properties normally available only with relatively thick holographic media. Possible benefits include substituting appropriate thin holographic media in applications for which no suitable thick holographic medium exists and using novel materials such as multiplequantum-well compound semiconductors to fabricate VHOE's having unique material properties.

As we discussed above, a VHOE in which a single grating is recorded yields a +1 -order diffraction response only at Bragg incidence. An SVHOE having the same total thickness can emulate a VHOE's +1-order diffraction response over an arbitrary angular range centered about the Bragg angle for that grating. The extent of the angular range is dependent on the normalized buffer-layer thickness of the SVHOE (and therefore on the number of modulation layers because the total thickness is fixed). For example, the +1-order angular sensitivity shown in Fig. 4(c) for an 11-layer SVHOE $\left(Q_{b} / 2 \pi=4.36\right)$ exhibits excellent emulation of the +1 -order VHOE diffraction response shown in Fig. 8 over an angular range of at least $1 \mathrm{deg}$ centered about the Bragg angle. Decreasing $Q_{b}$ (while increasing the number of layers to keep the total thickness constant) increases the angular emulation range.

The situation becomes more complicated when an SVHOE is used to emulate a VHOE in which multiple gratings are recorded. For emulation over a particular angular range, the buffer-layer thickness must be chosen such that no extraneous +1 -order response from any of the desired gratings occur within this range. In addition, if the desired grating strength is large enough to cause competition between overlapping +1 - and -1-order diffraction peaks, the buffer-layer thickness must further satisfy the condition that no -1-order response from any of the gratings overlaps with desired +1 -order responses within the angular emulation range. Otherwise, competition between +1 - and -1 -order diffraction responses can occur such that the fidelity of the diffracted beams is compromised. The necessity of excluding extraneous +1 -order diffraction responses and avoiding fidelity errors caused by -1-order overlap are critical design requirements in the use of SVHOE's as substitutes for traditional VHOE's.

\section{NORMALIZED BUFFER-LAYER THICKNESS EFFECTS AT BRAGG INCIDENCE}

Examination of SVHOE diffraction properties as a function of the normalized buffer-layer thickness $Q_{b}$ leads to the observation of further novel SVHOE diffraction behavior. ${ }^{15}$ In Subsection 4.A we show that the diffraction efficiency of the +1 order is periodic as a function of $Q_{b} / 2 \pi$ for readout at Bragg incidence and discuss the origins of this behavior. In Subsection 4.B we show how this behavior can be utilized to perform various notchfiltering functions. The following discussion assumes that the readout beam is incident at the Bragg angle.

\section{A. Periodic +1-Order Diffraction Behavior as a Function of $Q_{b} / 2 \pi$}

To illustrate the periodic dependence of the diffraction efficiency of the +1 order on the normalized buffer-layer thickness, we show in Fig. 13 the first-order diffraction efficiency of a five-layer SVHOE as a function of $Q_{b} / 2 \pi$

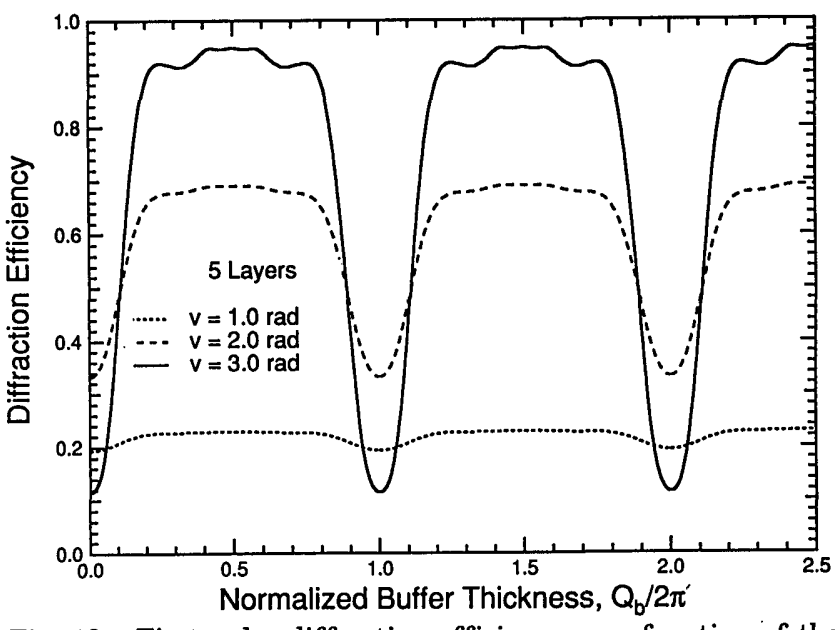

Fig. 13. First-order diffraction efficiency as a function of the normalized buffer-layer thickness $Q_{b} / 2 \pi$ for a five-layer SVHOE parameterized by the grating strength $v$. 
for several values of the total grating strength. At zero buffer-layer thickness, the diffraction efficiency corresponds to the limiting case of Raman-Nath diffraction at each grating strength (i.e., all the modulation layers are immediately adjacent to one another, and the SVHOE is thin enough that it operates in the Raman-Nath diffraction regime). As the normalized buffer-layer thickness increases from zero, the diffraction efficiency rapidly increases to nearly the values expected for a Bragg grating (recall that a five-layer SVHOE does not quite yield the same diffraction efficiency as a Bragg grating). However, as $Q_{b}$ continues to increase, the diffraction efficiency again becomes the same as a Raman-Nath grating at $Q_{b} / 2 \pi=$ 1.0. This behavior periodically repeats itself as the normalized buffer-layer thickness increases.

Note that instances of Raman-Nath diffraction efficiency occur at integer values of $Q_{b} / 2 \pi$, which according to Fig. 12 corresponds to -1 -order diffraction peaks being tuned through the +1 -order response that always occurs at Bragg incidence. Monitoring the other diffraction orders (including the zeroth order) reveals that they also exhibit Raman-Nath diffraction efficiencies for integer values of $Q_{b} / 2 \pi$. We note, however, that, despite the appearance of Raman-Nath diffraction efficiencies, the associated Raman-Nath property of insensitivity to the incidence angle of the readout beam is not present.

An alternative view of the curious occurrence of RamanNath diffraction efficiencies is shown in Fig. 14, in which the first-order diffraction efficiency is displayed as a function of total grating strength for $Q_{b} / 2 \pi$ equal to 0.5 (i.e., centered in a Bragg-regime region) and to 1.0 (i.e., at an occurrence of Raman-Nath diffraction efficiency). At $Q_{b} / 2 \pi=0.5$ the dependence of the diffraction efficiency on grating strength is close to the bulk grating result of $\sin ^{2}(v / 2){ }^{54}$ which it asymptotically approaches as the number of layers increases. At $Q_{b} / 2 \pi=1.0$, the diffraction efficiency is exactly the same as for a grating operating in the Raman-Nath regime, namely, $J_{1}^{2}(v),{ }^{55}$ in which $J_{1}$ is the first-order Bessel function of the first kind.

We can understand why Raman-Nath diffraction efficiencies occur at integer values of $Q_{b} / 2 \pi$ by considering the relative phases of all the diffraction orders as they propagate from modulation layer to modulation layer. Let $\phi_{m}$ be the relative phase difference between the 0 th and $m$ th diffraction orders after propagation between two SVHOE layers. This relative phase difference divided by $2 \pi$ can be written as [see Eq. (3)]

$$
\begin{aligned}
\phi_{m} / 2 \pi & =n d_{b}\left(\theta_{0}^{2}-\theta_{m}^{2}\right) /\left(2 \lambda_{0}\right) \\
& =m(\alpha-m / 2) Q_{b} / 2 \pi,
\end{aligned}
$$

in which $\theta_{m}$ is the angle at which the $m$ th diffraction order propagates (as shown in Fig. 15) and $\alpha$ is the normalized incidence angle defined above. Equation (9) is derived by realizing that $\theta_{m}$ can be expressed as $\theta_{m}=\theta_{0}-m \lambda / \Lambda$. Note that when $\alpha=1 / 2$ (Bragg incidence) and $Q_{b} / 2 \pi$ is an integer the relative phase difference $\phi_{m}$ is an integer times $2 \pi$ for every diffraction order. Thus the relative phase of all the diffraction orders is the same at each modulation layer. Propagation through the buffer regions therefore has no effect on the readout of the grating. The net result is that the grating recorded in the SVHOE appears to be a thin grating as experienced by the readout beam, and Raman-Nath diffraction efficiencies obtain for all the diffraction orders.

When one compares Figs. 13 and 14 it is apparent that the depth of each notch in Fig. 13 is simply the difference between the diffraction efficiency curves shown in Fig. 14 at the appropriate grating strength. Note that at a grating strength of $3.83 \mathrm{rad}$ (i.e., at the first Raman-Nath diffraction null) the diffraction efficiency in the center of the notches should therefore go to zero. Figure 16 shows the first-order diffraction efficiency as a function of $Q_{b} / 2 \pi$ at this grating strength for SVHOE's having two, five, and twenty-one modulation layers. In addition to there being zero diffraction efficiency at the bottom of each notch, this figure shows how the notch width narrows as the number

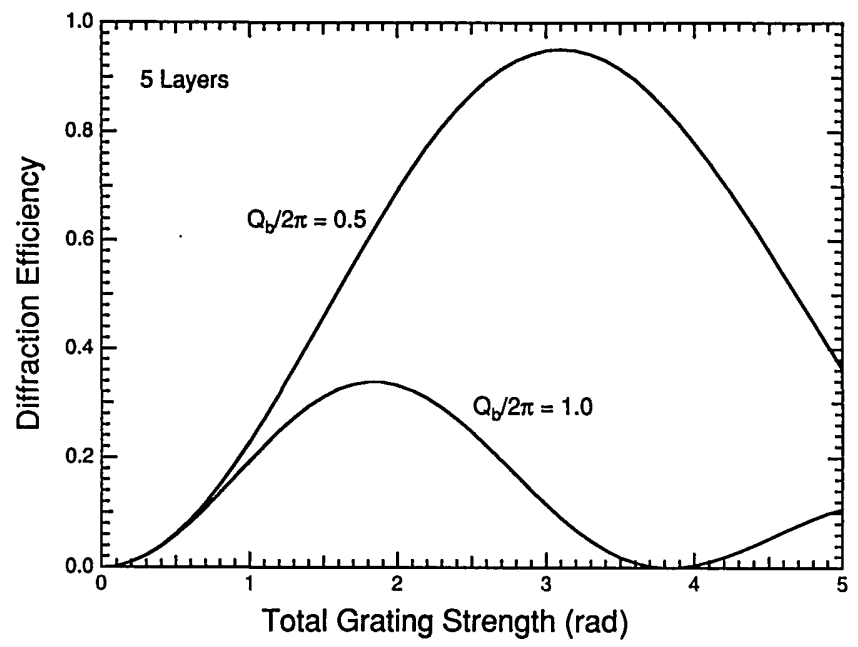

Fig. 14. First-order diffraction efficiency as a function of grating strength for a five-layer SVHOE for different values of the normalized buffer-layer thickness $Q_{b} / 2 \pi$.

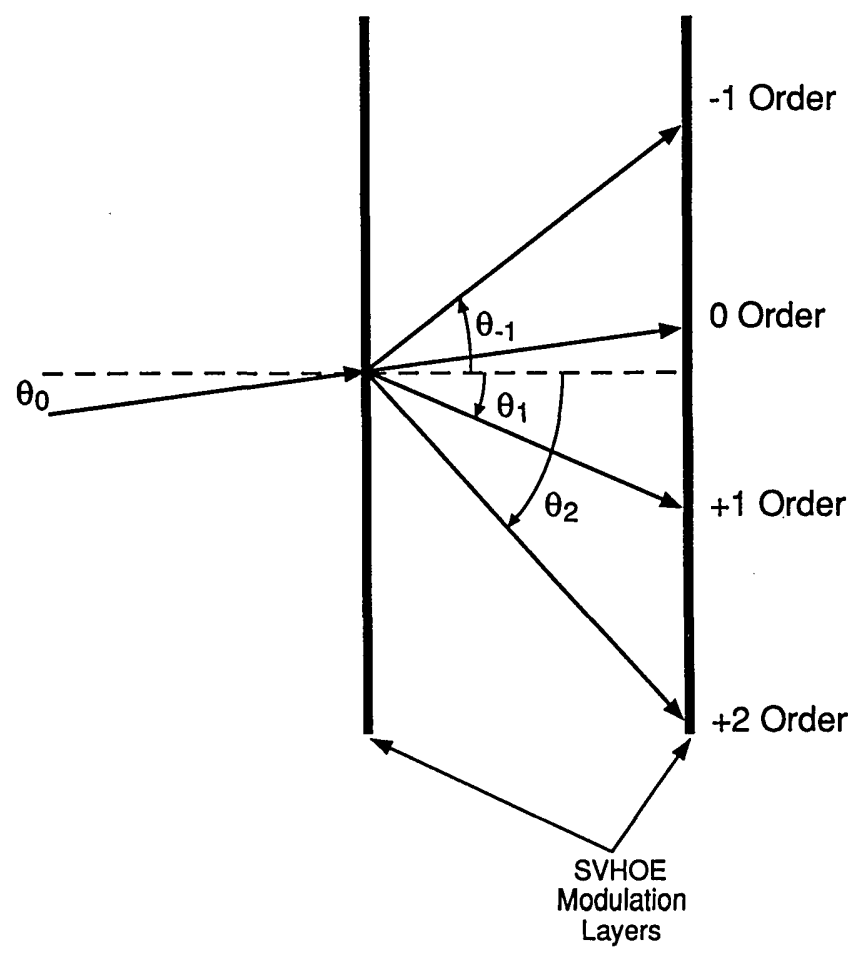

Fig. 15. Propagation angles of the -1 to +2 diffraction orders between two SVHOE modulation layers. 


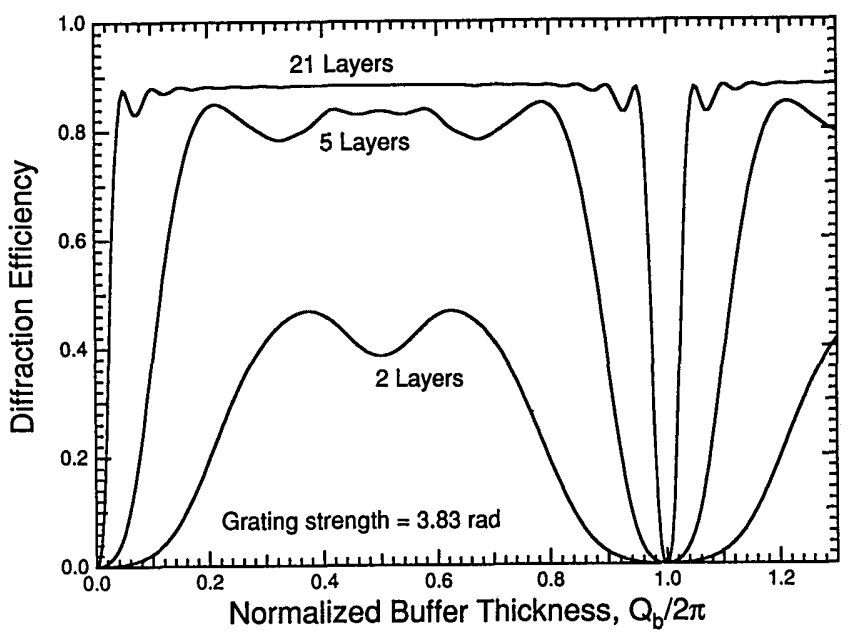

Fig. 16. First-order diffraction efficiency as a function of the normalized buffer-layer thickness $Q_{b} / 2 \pi$ for different numbers of modulation layers. The total grating strength in each case is chosen as $3.83 \mathrm{rad}$, which results in zero diffraction efficiency at the center of each notch.

layers increases. For more than two or three layers, the FWHM of the notches is approximated quite well by $1 /(N-1)$ in units of $Q_{b} / 2 \pi$.

\section{B. SVHOE Notch Filtering}

The availability of notched SVHOE diffraction behavior suggests the potential of performing various notchfiltering functions if the physical quantity to be filtered can be mapped to this behavior. Because the normalized buffer-layer thickness $Q_{b}$ is a function of buffer-layer thickness, grating period, and readout wavelength, the first diffraction order exhibits notched diffraction behavior as a function of any of these parameters when the others are held constant and Bragg incidence is maintained.

\section{Wavelength Notch Filtering}

As an example, consider the diffraction response of a 21-layer SVHOE as a function of readout wavelength as shown in Fig. 17. The readout beam is assumed to be Bragg incident at each wavelength. For simplicity, the refractive-index modulation is taken as a constant for all wavelengths; it is set such that the grating strength is $3.83 \mathrm{rad}$ at a wavelength of $0.80 \mu \mathrm{m}$. The other SVHOE parameters are chosen such that $Q_{b} / 2 \pi$ is an integer at this wavelength. As is seen in Fig. 17, the diffraction efficiency of the +1 order shows a distinctly notched behavior as a function of the wavelength of the incident readout beam. Note that the notch centered at a wavelength of $0.80 \mu \mathrm{m}$ has zero diffraction efficiency at the center of the notch. This suggests the possibility of using an SVHOE as a narrow-band wavelength notch filter. In Fig. 17 the notch widths (FWHM) are $4 \mathrm{~nm}$. In general, the spectral width of each notch is given by $\Delta \lambda /(N-1)$, in which $\Delta \lambda$ is the spectral separation of the notches and is equal to $n \Lambda^{2} / d_{b}$. By increasing the buffer-layer thickness of the SVHOE in Fig. 17 by a factor of 13 and increasing the number of layers to 61 , one can decrease the spectral width of the notches to $0.1 \mathrm{~nm}$.

Another intriguing possibility is the construction of an
SVHOE-based tunable wavelength notch filter by varying any of the parameters on which the normalized bufferlayer thickness depends, including the refractive index of the buffer layers. For example, it may be possible to fabricate an electrically tunable SVHOE-based wavelength notch filter by incorporating an electro-optic material (such as a liquid crystal) in the buffer-layer regions of the SVHOE.

Several other features of the diffraction behavior shown in Fig. 17 deserve mention. First, even though we have assumed that the refractive-index modulation is constant for all wavelengths, the grating strength itself is inversely proportional to the wavelength of the readout beam. This grating strength dispersion manifests itself as a slowly varying envelope that sets the overall shape of the diffraction response seen in Fig. 17. (By overall shape we mean the general shape of the curve without the notches and the ringing that occurs at some of the notch edges.) Second, there is significant ringing at the edges of the two leftmost notches. This is due to overmodulation of the grating

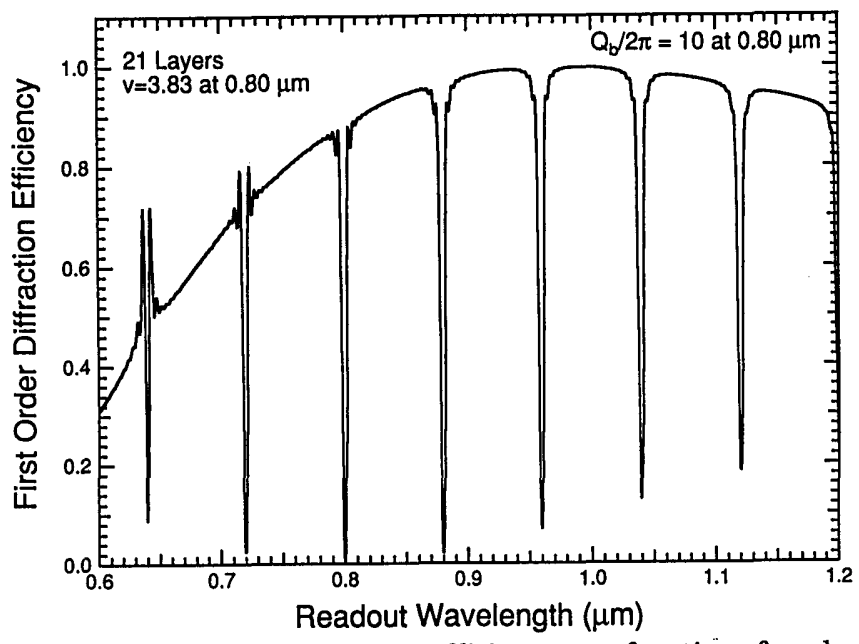

Fig. 17. First-order diffraction efficiency as a function of readout beam wavelength for a 21-layer SVHOE. Bragg incidence is assumed at each wavelength.

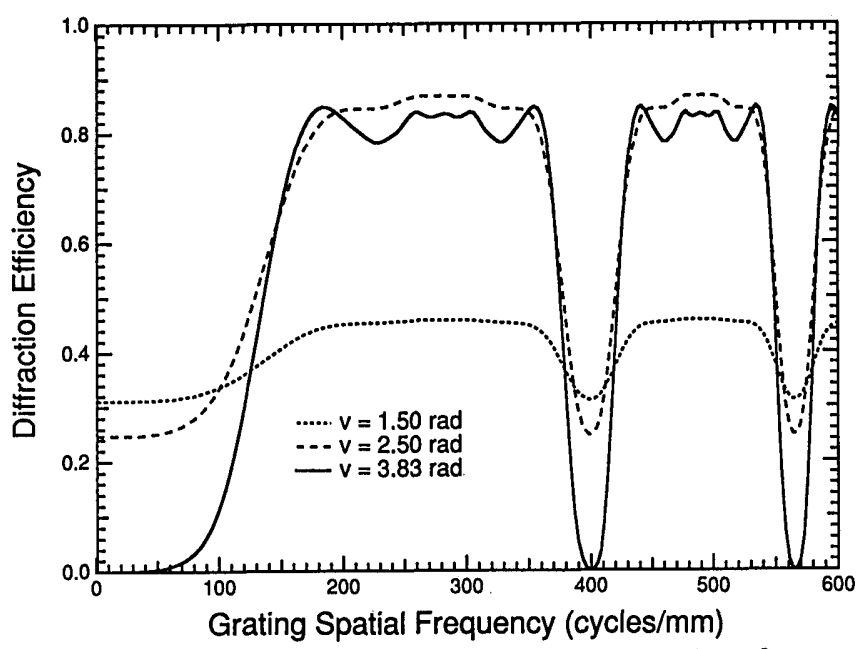

Fig. 18. First-order diffraction efficiency as a function of grating spatial frequency for a five-layer SVHOE for different values of the total grating strength $v$. Bragg incidence is assumed at each grating spatial frequency. 
(i.e., $v>\pi$ ) and is not a numerical artifact of the BPM simulation used to obtain the curve.

\section{Grating Spatial Frequency Notched Diffraction Response}

A further example of the notched diffraction response of the +1 order is illustrated in Fig. 18. Here the diffraction efficiency of a five-layer SVHOE is shown as a function of the grating spatial frequency for several values of the total grating strength. Bragg incidence is assumed at each grating spatial frequency. The readout wavelength, refractive index, and buffer-layer thickness are chosen such that $Q_{b} / 2 \pi=1$ at 400 cycles $/ \mathrm{mm}$. Note that, when the total grating strength is $3.83 \mathrm{rad}$, specific grating spatial frequencies exist that result in complete extinction of the diffracted first order for readout at Bragg incidence. However, this does not imply that the readout beam propagates through the SVHOE undisturbed. Rather, the SVHOE acts as a thin grating with a grating strength of $3.83 \mathrm{rad}$ and therefore diffracts light into higher orders (with some of the light remaining in the zeroth order).

The notches in diffraction efficiency occur at grating spatial frequencies of $\left(l_{s} n / \lambda d_{b}\right)^{1 / 2}$ (in which $l_{s}$ is an integer greater than zero), whereas the FWHM of each notch is $n \Lambda /\left(2 \lambda D_{t}\right)$. Note that changing the buffer-layer thickness $d_{b}$ changes the notch positions. An intriguing possibility thus exists for an SVHOE to be constructed by using modulation layers having an optically or electrically controlled grating strength or recording sensitivity. By turning on and off various sets of modulation layers, one can change the effective buffer-layer thickness such that the position of the grating spatial frequency notches can be varied. ${ }^{13,16}$ This permits a novel tailoring of the SVHOE grating spatial frequency sensitivity. The use of electrically or optically controlled modulation layers is a further SVHOE design flexibility that can be utilized to change other SVHOE diffraction properties (such as the angular sensitivity of the \pm 1 orders) in addition to the grating spatial frequency dependence of the +1 order.

\section{SUMMARY}

We have discussed the dependence of the periodic \pm 1 -order SVHOE angular sensitivity on buffer-layer thickness, number of layers, and total SVHOE thickness. This behavior can be explained by examining the relative phases of the zeroth order and the respective +1 or -1 order at each modulation layer. We have shown that at Bragg incidence a novel periodic notched diffraction behavior of the first order occurs as a function of the normalized bufferlayer thickness. Notches occur at integer values of the normalized thickness parameter $Q_{b} / 2 \pi$ (corresponding to exact overlap of the \pm 1 -order angular sensitivities). At integer values of $Q_{b} / 2 \pi$, the diffraction efficiency of each order becomes the same as a single thin grating of equivalent total grating strength because of the relative phasing of the diffraction orders as they propagate between the modulation layers. A possible application of this phenomenon is narrow-band wavelength notch filtering. Utilizing the SVHOE concept, one can assemble thin holographic materials into structures designed to access unique SVHOE diffraction properties. Such structures can be used either as the basis for novel devices as described herein or to emulate VHOE properties over particular parameter ranges.

\section{ACKNOWLEDGMENTS}

This research was supported in part by the Defense Advanced Research Projects Agency. G. Nordin gratefully acknowledges support from a Hughes Doctoral Fellowship and a Center for Photonic Technology Prize Fellowship from the University of Southern California.

*Present address, Department of Electrical and Computer Engineering, The University of Alabama in Huntsville, Huntsville, Alabama 35899.

${ }^{\dagger}$ Present address, Crystal Technology, Inc., 1040 East Meadow Circle, Palo Alto, California 94303.

\section{REFERENCES}

1. K. Wagner and D. Psaltis, "Multilayer optical learning networks," Appl. Opt. 28, 5061-5074 (1987).

2. D. Z. Anderson and D. M. Lininger, "Dynamic optical interconnects: volume holograms as optical two-port operators," Appl. Opt. 26, 5031-5038 (1987).

3. D. Psaltis, D. Brady, X.-G. Gu, and K. Hsu, "Optical implementation of neural computers," in Optical Processing and Computing, H. Arsenault, ed. (Academic, San Diego, Calif., 1988).

4. D. Psaltis, D. J. Brady, and K. Wagner, "Adaptive optical networks using photorefractive crystals," Appl. Opt. 27, 17521758 (1988).

5. E. G. Paek, J. R. Wullert, and J. S. Patel, "Holographic implementation of a learning machine based on a multicategory perceptron algorithm," Opt. Lett. 14, 1303-1305 (1989).

6. J. H. Hong, S. Campbell, and P. Yeh, "Optical pattern classifier with perceptron learning," Appl. Opt. 29, 3019-3025 (1990).

7. P. Asthana, G. Nordin, S. Piazzolla, A. R. Tanguay, Jr., and B. K. Jenkins, "Analysis of interchannel crosstalk and throughput efficiency in highly multiplexed fan-out/fan-in holographic interconnections," in Annual Meeting, Vol. 15 of 1990 OSA Technical Digest Series (Optical Society of America, Washington, D.C., 1990), p. 242.

8. D. L. Staebler, W. J. Burke, W. Phillips, and J. J. Amodei, "Multiple storage and erasure of fixed holograms in Fe-doped lithium niobate," Appl. Phys. Lett. 26, 182-184 (1975).

9. F. H. Mok, M. C. Tackitt, and H. M. Stoll, "Storage of 500 high-resolution holograms in a $\mathrm{LiNbO}_{3}$ crystal," Opt. Lett. 16, 605-607 (1991).

10. A. M. Glass, D. D. Nolte, D. H. Olson, G. E. Doran, D. S. Chemla, and W. H. Knox, "Resonant photodiffractive fourwave mixing in semi-insulating GaAs/AlGaAs quantum wells," Opt. Lett. 15, 264-266 (1990).

11. A. Partovi, A. M. Glass, D. H. Olson, G. J. Zydik, K. T. Short, R. D. Feldman, and R. F. Austin, "High sensitivity optical image processing device based on CdZnTe/ZnTe multiple quantum well structures," Appl. Phys. Lett. 59, 1832-1834 (1991).

12. A. R. Tanguay, Jr., and R. V. Johnson, "Stratified volume holographic optical elements," J. Opt. Soc. Am. A 3(13), P53 (1986).

13. R. V. Johnson and A. R. Tanguay, Jr., "Stratified volume holographic optical elements," Opt. Lett. 13, 189-191 (1988).

14. R. V. Johnson and A. R. Tanguay, Jr., "Stratified volume holographic optical elements," Opt. News 14(12), 30-31 (1988).

15. G. P. Nordin, R. V. Johnson, and A. R. Tanguay, Jr., "Physical characterization of stratified volume holographic optical elements," in Annual Meeting, Vol. 11 of 1988 OSA Technical Digest Series (Optical Society of America, Washington, D.C., 1988), p. 106.

16. L. Domash, J. Schwartz, A. Nelson, and P. Levin, "Active holographic interconnects for interfacing volume storage," in Image Storage and Retrieval Systems, A. Jamberdino, and W. Niblack, eds., Proc. Soc. Photo-Opt. Instrum. Eng. 1662, 211-218 (1991).

17. J. A. Fleck, J. R. Morris, and M. D. Feit, "Time-dependent propagation of high energy laser beams through the atmosphere," Appl. Phys. 10, 129-160 (1976). 
18. D. Yevick and L. Thylen, "Analysis of gratings by the beampropagation method," J. Opt. Soc. Am. 72, 1081-1089 (1982).

19. R. V. Johnson and A. R. Tanguay, Jr., "Optical beam propagation method for birefringent phase grating diffraction," Opt. Eng. 25, 235-249 (1986).

20. M. D. Feit and J. A. Fleck, Jr., "Light propagation in gradedindex optical fibers," Appl. Opt. 17, 3990-3998 (1978).

21. L. Thylen and D. Yevick, "Beam propagation method in anisotropic media," Appl. Opt. 21, 2751-2754 (1982).

22. H. J. Caulfield, D. H. McMahon, and R. A. Soref, "Stacked hologram apparatus," U.S. patent 3,635,538 (January 18, 1972).

23. H. J. Caulfield, "Stacked page oriented holographic memory," in Holographic Optics: Design and Applications, I. Cindrich, ed., Proc. Soc. Photo-Opt. Instrum. Eng. 883, 203-206 (1988).

24. D. Pohl, "Stacked optical memories," Appl. Opt. 13, 341-346 (1974).

25. J. B. Thaxter and M. Kestigian, "Unique properties of SBN and their use in a layered optical memory," Appl. Opt. 13, 913-924 (1974).

26. K. Chalasinska-Macukow, B. Karczewski, and T. Szoplik, "Reconstruction of two stacked Fourier holograms," Opt. Commun. 27, 311-316 (1978)

27. K. Chalasinska-Macukow and T. Szoplik, "Reconstruction of two stacked Fourier holograms-experiments and results," Opt. Commun. 33, 245-250 (1980).

28. J. Slaby, T. Szoplik, and K. Chalasinska-Macukow, "Cascaded phase Fourier holograms," Opt. Acta 30, 529-543 (1983)

29. J. Slaby and T. Szoplik, "Resonant modes in a cascaded system of Fourier holograms," Opt. Acta 33, 301-313 (1986).

30. D. J. De Bitetto, "On the intensifying property of a pile-ofgratings," Appl. Opt. 9, 59-61 (1970).

31. L. E. Hargrove, "Diffraction of light passing through two adjacent ultrasonic progressive waves of different frequency," J. Acoust. Soc. Am. 32, 940 (1960).

32. L. E. Hargrove, E. A. Hiedemann, and R. Mertens, "Diffraction of light by two spatially separated parallel ultrasonic waves of different frequency," Z. Phys. 167, 326-336 (1962).

33. P. Kwiek, "Light diffraction by two spatially separated ultrasonic waves," J. Acoust. Soc. Am. 86, 2261-2272 (1989).

34. V. N. Malysh, O. I. Ovcharenko, and A. N. Osovitskii, "Light diffraction by a layered structure with periodically modulated interfaces," Opt. Spectrosc. (USSR) 58, 513-516 (1985).

35. K. Kodate, T. Kamiya, and M. Kamiyama, "Double diffraction in the Fresnel region," Jpn. J. Appl. Phys. 10, 1040-1045 (1971).

36. K. Kodate, T. Kamiya, H. Takenaka, and H. Yanai, "Double diffraction of phase gratings in the Fresnel region," Jpn. J. Appl. Phys. 14, 1323-1334 (1975).

37. D. A. Larson, T. D. Black, M. Green, R. G. Torti, Y. J. Wang, and R. Magnusson, "Optical modulation by a traveling sur- face acoustic wave and a holographic reference grating," J. Opt. Soc. Am. A 7, 1745-1750 (1990).

38. F. Calligaris, P. Ciuti, and I. Gabrielli, "Temporal light modulation in thick-screen diffraction by ultrasound beam plus amplitude grating," J. Acoust. Soc. Am. 61, 959-964 (1977).

39. V. A. Komotskii and V. F. Nikulin, "Theoretical analysis of diffraction of a Gaussian optical beam by a system of two diffraction gratings," Opt. Spectrosc. (USSR) 63, 239-242 (1987).

40. A. F. Bessonov, L. N. Deryugin, and V. A. Komotskii, "Phenomena of optical wave diffraction by traveling spatial phase modulation on stationary phase gratings," Opt. Spectrosc. (USSR) 49, 81-84 (1980).

41. V. A. Komatskii and T. D. Black, "Analysis and application of stationary reference grating method for optical detection of surface acoustic waves," J. Appl. Phys. 51, 129-136 (1981).

42. B. Y. Zel'dovich and T. V. Yakovleva, "Theory of a two-layer hologram," Sov. J. Quantum. Electron. 14, 323-328 (1984).

43. B. Y. Zel'dovich, D. I. Mirovitskii, N. V. Rostovtseva, and O. B. Serov, "Characteristics of two-layer phase holograms," Sov. J. Quantum. Electron. 14, 364-369 (1984).

44. N. N. Evtikhiev, D. I. Mirovitskiy, N. V. Rostovtseva, O. B. Serov, T. V. Yakovleva, and B. Y. Zel'dovich, "Bilayer holograms: theory and experiment," Opt. Acta 33, 255-268 (1986).

45. N. N. Evtikhiev, D. I. Mirovitskii, N. V. Rostovtseva, and O. B. Serov, "Multilayer holographic functional element in an analog-digital converter," Sov. J. Quantum. Electron. 16, 1180-1184 (1987).

46. M. Kujawinska, "Fresnel-field analysis of double-grating systems and their application in phase-stepping grating interferometers," J. Opt. Soc. Am. A 5, 849-857 (1988).

47. M. C. Gupta, "Diffraction of a light beam by doubly periodic structures," Opt. Lett. 16, 1301-1303 (1991).

48. M. Kujawinska, "Development of the theory of quasi-periodic diffraction grating systems," J. Opt. Soc. Am. A 5, 206-213 (1988).

49. A. P. Yakimovich "Multilayer three-dimensional holographic gratings," Opt. Spectrosc. (USSR) 49, 85-88 (1980).

50. H. Blok and G. Mur, "Diffraction by a double grating," Appl. Sci. Res. 26, 389-397 (1972).

51. S.-W. Lee, G. Zarrillo, and C.-L. Law, "Simple formulas for transmission through periodic metal grids or plates," IEEE Trans. Antennas Propag. AP-30, 904-909 (1982).

52. R. Petit and G. Tayeb, "Theoretical and numerical study of gratings consisting of periodic arrays of thin and lossy strips," J. Opt. Soc. Am. A 7, 1686-1692 (1990).

53. R. J. Collier, C. B. Burckhardt, and L. H. Lin, Optical Holography (Academic, New York, 1971).

54. H. Kogelnik, "Coupled wave theory for thick hologram gratings," Bell Syst. Tech. J. 48, 2909-2947 (1969).

55. J. W. Goodman, Introduction to Fourier Optics (McGrawHill, San Francisco, Calif., 1968). 